\title{
Surgical Trial in Lobar Intracerebral Haemorrhage (STICH II) Protocol
}

\author{
A David Mendelow ${ }^{1 *}$, Barbara A Gregson ${ }^{1}$, Patrick M Mitchell1', Gordon D Murray², Elise N Rowan ${ }^{1}$, Anil R Gholkar ${ }^{3}$ \\ and for the STICH II Investigators
}

\begin{abstract}
Background: Within the spectrum of spontaneous intracerebral haemorrhage there are some patients with large or space occupying haemorrhage who require surgery for neurological deterioration and others with small haematomas who should be managed conservatively. There is equipoise about the management of patients between these two extremes. In particular there is some evidence that patients with lobar haematomas and no intraventricular haemorrhage might benefit from haematoma evacuation. The STICH II study will establish whether a policy of earlier surgical evacuation of the haematoma in selected patients will improve outcome compared to a policy of initial conservative treatment.
\end{abstract}

Methods/Design: an international multicentre randomised parallel group trial. Only patients for whom the treating neurosurgeon is in equipoise about the benefits of early craniotomy compared to initial conservative treatment are eligible. All patients must have a CT scan confirming spontaneous lobar intracerebral haemorrhage $(\leq 1 \mathrm{~cm}$ from the cortex surface of the brain and 10-100 $\mathrm{ml}$ in volume). Any clotting or coagulation problems must be corrected and randomisation must take place within 48 hours of ictus. With 600 patients, the study will be able to demonstrate a $12 \%$ benefit from surgery $(2 p<0.05)$ with $80 \%$ power.

Stratified randomisation is undertaken using a central 24 hour randomisation service accessed by telephone or web. Patients randomised to early surgery should have the operation within 12 hours. Information about the status (Glasgow Coma Score and focal signs) of all patients through the first five days of their trial progress is also collected in addition to another CT scan at about five days (+/- 2 days). Outcome is measured at six months via a postal questionnaire to the patient. Primary outcome is death or severe disability defined using a prognosis based 8 point Glasgow Outcome Scale. Secondary outcomes include: Mortality, Rankin, Barthel, EuroQol, and Survival.

Trial Registration: ISRCTN: ISRCTN22153967

\section{Trial Hypothesis}

A policy of earlier surgical evacuation of the haematoma in selected patients with spontaneous lobar ICH improves outcome compared to a policy of initial conservative treatment.

\section{Background}

The role of operative neurosurgical intervention in intracerebral haemorrhage is controversial

Spontaneous intracerebral haemorrhage (ICH) accounts for 10 to $40 \%$ of all cases of stroke (there is some

\footnotetext{
* Correspondence: a.d.mendelow@ncl.ac.uk

'STICH Office, Neurosurgical Trials Unit, Newcastle University, 3-4 Claremont Terrace, Newcastle upon Tyne, NE2 4AE, UK

Full list of author information is available at the end of the article
}

variation between countries) and is common in younger patients [1]. The morbidity and mortality exceed $60 \%$ and young disabled survivors are a significant burden to both Health and Social Services with only $12 \%$ of all ICH patients emerging with minor handicap [2]. The role of operative neurosurgical intervention is controversial and the practice continues to be haphazard $[3,4]$. Within the spectrum of ICH there are some patients (with large or space occupying $\mathrm{ICH}$ ) who require surgery for neurological deterioration and others with small haematomas who should be managed conservatively. There is equipoise about the management of patients between these two extremes. Some patients have a penumbra of functionally impaired but potentially viable

\section{Biomed Central}


tissue around the ICH. Surgical removal of the clot may improve the function and recovery in this penumbra [5].

\section{Findings from previous randomised trials have not been significant}

The first randomised trial of Surgical Treatment of $\mathrm{ICH}$, published in 1961 [6] did not show a significant advantage for either surgical or conservative treatment. However this trial was prior to $\mathrm{CT}$ and modern operative techniques and care facilities. Between 1989 and 1992 results from four small prospective randomised trials were published. Two trials showed a non-significant advantage for surgery $[7,8]$ and two favoured conservative treatment but the advantage was not significant $[9,10]$. Two further very small trials have been published both showing a non-significant advantage in favour of surgery $[11,12]$. Each of these reported problems with recruiting sufficient patients from a single centre and argued for the importance of a large randomised multicentre trial. Further trials have reported since 2000: a large trial of 500 patients showing a non-significant advantage for surgery [13]; two smaller trials showing a significant advantage for surgery $[14,15]$ and a small trial suggesting an advantage for conservative treatment [16]. The need to gain robust evidence to support clinical decision making led to the initiation of the Surgical Trial in Intracerebral Haemorrhage (STICH). The funding for STICH was provided from the UK by the MRC and the Stroke Association and was activated in 1998 and 1995 respectively. This trial is the largest to date and successfully recruited 1033 patients from 87 centres around the world. It also suggested a small non-significant advantage for surgery [17].

\section{Meta-analyses suggest that surgery may benefit a sub group of patients}

A meta-analysis of the first four published randomised controlled trials was conducted by Prasad et al (2000) for the Cochrane Collaboration [18]. This was updated in 2006 (see figure 1) to include all twelve trials published prior to 2006. Including all twelve trials gave an odds ratio of $0.85(\mathrm{CI} 0.71,1.02$ ) in favour of surgical treatment when the unfavourable outcome was death and an odds ratio of 0.86 (CI $0.72,1.03$ ) for the 11 trials with published data when the unfavourable outcome was severe disability or death.

Further detailed analysis of the CT images showed that $42 \%$ of patients included in STICH who had assessable scans also had an associated intraventricular haemorrhage (IVH). The prognosis for patients with intraventricular haemorrhage with or without hydrocephalus is much worse than that for intracerebral haemorrhage alone. Removing these patients from the analysis and focusing on superficial haematomas presented a more encouraging picture for surgery. There were 223 patients in STICH with such haematomas and with initial conservative treatment $37 \%$ achieved a favourable outcome using the prognosis based outcome methodology used in STICH [19]. By contrast $49 \%$ of patients achieved a favourable outcome with early surgery $(\mathrm{p}=0.080)$. Furthermore using prognosis based Rankin as the outcome variable a significant benefit was observed for

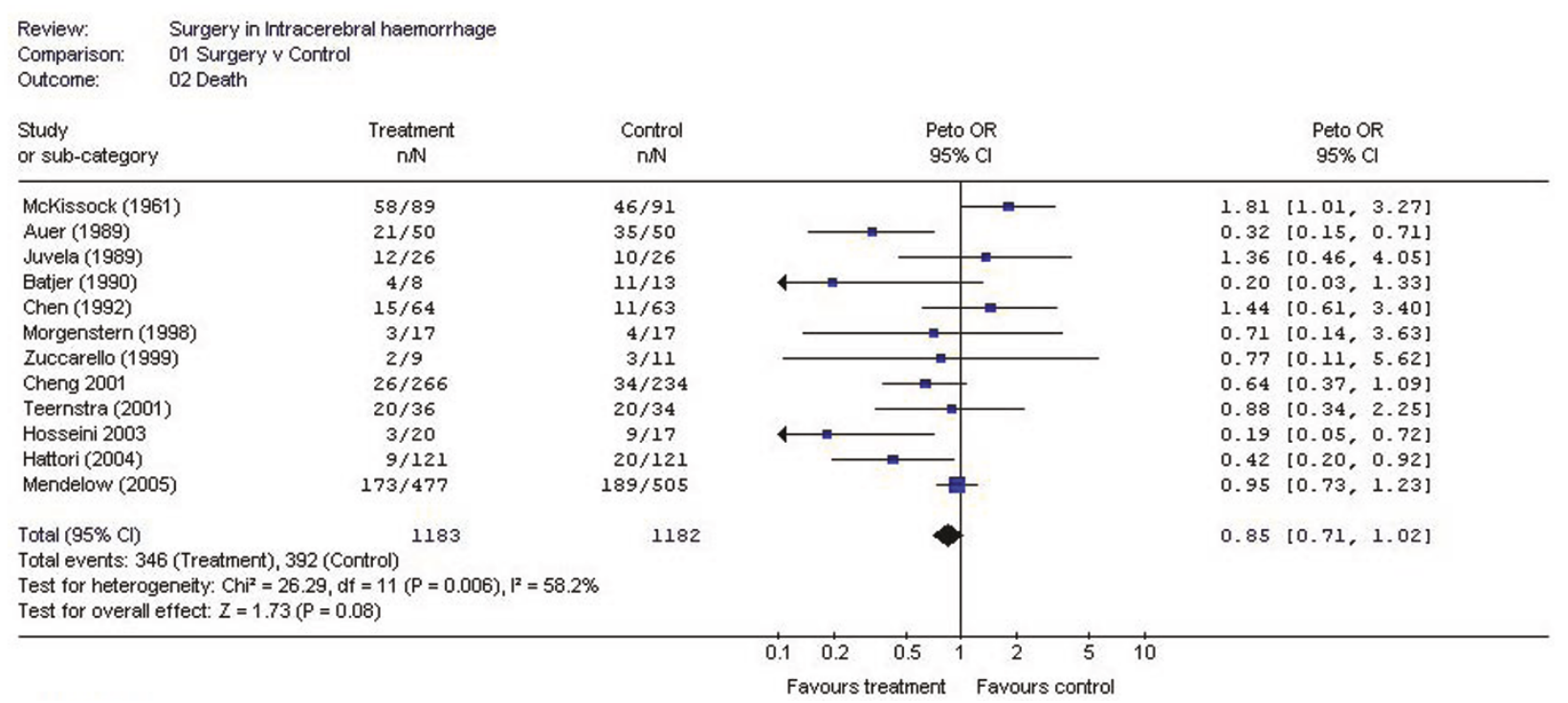

Figure 1 Meta-analysis of all surgical intracerebral haemorrhage trials (Poor outcome = death). 
surgical patients in this subgroup. $(\mathrm{p}=0.013)$. Although this is a post hoc identified subgroup, the exclusion of IVH makes clinical sense in the context of debulking surgery for lobar haematomas. The treatment of IVH is different and does not involve craniotomy.

The majority of patients in the other trials reported in the meta-analysis had deep haematomas. Only in the trials by Auer et al. (45 patients) and Teernstra et al. (23 patients) $[7,16]$ did the numbers with lobar $\mathrm{ICH}$ reach double figures. In the Auer et al. trial $54 \%$ of the 24 surgical patients had a favourable outcome compared to $29 \%$ of the 21 conservative patients. In the Teernstra trial $25 \%$ of the 16 surgical patients and $22 \%$ of the 9 conservative patients had a favourable outcome. Thus overall $42 \%$ of surgical patients and $27 \%$ of conservative patients had a favourable outcome. Figures $2 \mathrm{a}$ and $2 \mathrm{~b}$ show meta-analyses of lobar haematomas from these trials excluding and including the STICH study data.

Therefore the few published randomised controlled trial data that did exist concerning lobar haematomas supported the hypothesis that this subgroup might benefit from early surgery.
An unfortunate outcome of STICH had been that many people misinterpreted the results to argue that there was no need to operate on patients with $\mathrm{ICH}$ at all. However neurosurgeons know that early removal of an intracranial haemorrhage is highly effective postoperatively and in the context of trauma (extradural haematoma [20], and acute subdural haematoma [21]). It seems unlikely that surgery would be of benefit in one scenario and not in the other. To leave patients with lesions that should be removed (an unfortunate misinterpretation of STICH) would condemn such patients to non-operative treatment perhaps for evermore. Since STICH was not powered sufficiently to answer the question about this subgroup alone there was an urgent need to undertake STICH II.

\section{STICH II will establish whether surgery is of benefit to a sub group}

The trial aims to establish whether a policy of earlier surgical evacuation of the haematoma in selected patients with spontaneous lobar ICH will improve outcome compared to a policy of initial conservative

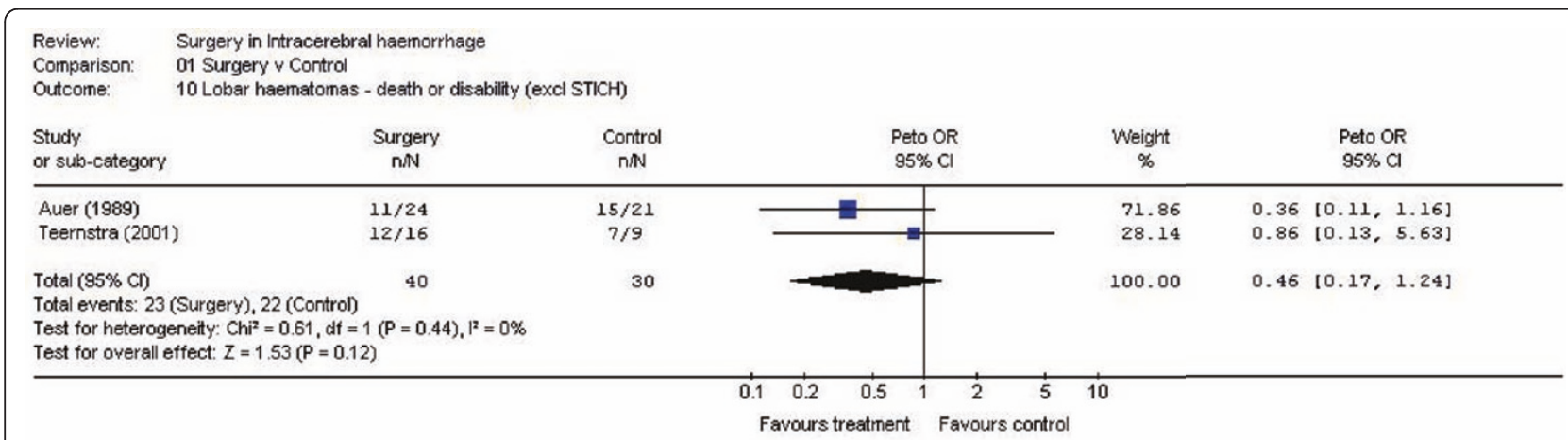

a) Meta-analysis of lobar haematomas only from trials (excluding STICH)

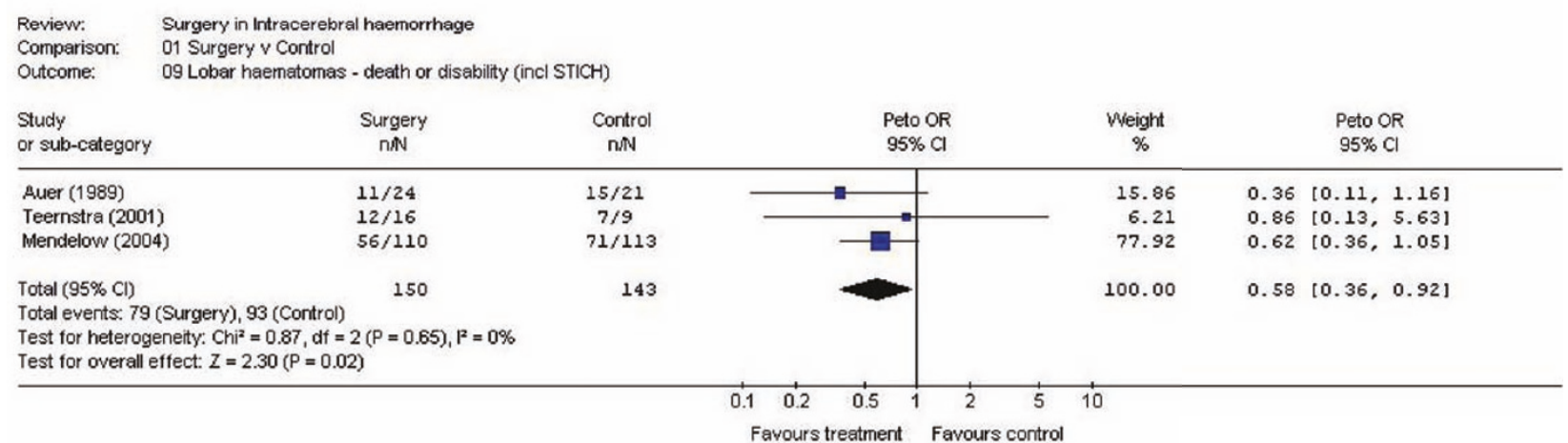

b) Meta-analysis of lobar haematomas only from trials (including STICH)

Figure 2 Meta-analysis of lobar haematomas only; a) excluding STICH, b) including STICH. 
treatment. The trial will also help to better define the indications for early surgery.

This will overcome two of the criticisms of STICH (timing was too late and sometimes location was too deep). The subgroup identified in STICH is clinically sensible and the hypothesis identified for STICH II is in line with current neurosurgical opinion.

\section{Design}

STICH II is an international multicentre randomised parallel group trial comparing early craniotomy to evacuate the haematoma with initial conservative treatment, following spontaneous superficial intracerebral haemorrhage affecting the lobar region only. Only patients for whom the treating neurosurgeon is in equipoise about the benefits of early craniotomy compared to initial conservative treatment are eligible for the trial. Outcome is measured at six months via a postal questionnaire including the Glasgow Outcome scale, Modified Rankin Scale, EuroQol and Barthel. Six hundred patients will be recruited to the trial.

\section{Centre eligibility}

At least 100 centres from around the world are included (UK, USA, Australia, Armenia, Czech Republic, Egypt, Germany, Greece, Hungary, India, Italy, Latvia, Lithuania, Macedonia, Mexico, Nepal, Pakistan, Poland, Romania, Russia, Spain, and Turkey). Only centres that can demonstrate effective trial experience and previous adherence to trial guidelines with high follow-up rates are eligible to take part.

\section{Approval to start}

MREC approval for the study was obtained from Scotland A Multicentre Research Ethics Committee. Appropriate local ethical approval is sought from each participating centre in the study with proof of the approval forwarded to the trial coordinating office before recruitment can be started. The trial is conducted according to local ethical and Research and Development procedures. An agreement is signed between the sponsor (Newcastle upon Tyne NHS Hospitals Foundation Trust), the holder of the study funding (Newcastle University) and the hospital centre prior to commencing the study at the centre.

\section{Inclusion Criteria}

- Evidence of a spontaneous lobar ICH on CT scan (1 $\mathrm{cm}$ or less from the cortex surface of the brain).

- Patient within 48 hours of ictus.

- Best MOTOR score on the Glasgow Coma Scale (GCS) of 5 or 6 and best EYE score on the GCS of 2 or more.
- Volume of haematoma between 10 and $100 \mathrm{ml}$ [Calculated using $(\mathrm{a} \times \mathrm{b} \times \mathrm{c}) / 2$ method].

\section{Exclusion Criteria}

- Clear evidence that the haemorrhage is due to an aneurysm or angiographically proven arteriovenous malformation.

- Intraventricular haemorrhage of any sort.

- ICH secondary to tumour or trauma.

- Basal ganglia, thalamic, cerebellar or brainstem haemorrhage or extension of a lobar haemorrhage into any of these regions.

- Severe pre-existing physical or mental disability or severe co-morbidity which might interfere with assessment of outcome.

- If surgery cannot be performed within 12 hours.

- If the haematological effects of any previous anticoagulants are not completely reversed.

\section{Trial interventions}

The trial intervention is early evacuation of the haematoma by the method preferred by the treating neurosurgeon, usually craniotomy, combined with appropriate best medical treatment versus best medical treatment, combined with delayed evacuation only if it becomes necessary later. In STICH $26 \%$ of patients crossed over from conservative treatment to surgery usually because of deterioration but information was scarce. This is a major problem with surgical trials and crossovers of this size are common [22]. In STICH II the aim is to have fewer crossovers and more detailed information about the reasons. Further information about the status (GCS and focal signs) of all patients through the first five days of their trial progress is collected in order to be able to monitor the change in status that leads to a change in equipoise for the treating neurosurgeon. All patients also have an additional CT scan at about five days (+/2 days) to assess changes in the haematoma size with and without surgery. This will enable the study to demonstrate the amount of clot removed by surgery.

\section{Allocation of patients and consent}

All patients who are considered for STICH II must have a CT scan to confirm the diagnosis and the size and location of the haematoma. Any clotting or coagulation problems must be corrected.

Written witnessed informed consent of patients must be obtained prior to randomisation by trained neurosurgical staff. A member of neurosurgical staff must provide each patient and their relatives with a written information sheet about the study and allow as much time as possible to 
discuss the options. If the patient is unable to give consent themselves due to the nature of the haemorrhage, a personal representative must be approached to give assent on behalf of the patient. The personal representative is the person with the closest personal relationship with the patient who is themselves capable and willing to assent on behalf of the patient. If the patient is unable to consent and the closest relative is not available the patient cannot be included in the study. In Scotland, if proxy consent is necessary this is obtained from the welfare guardian or, if there is none, from the nearest relative.

One copy of the signed consent/assent form is given to the patient, one is filed in the patient notes and one is filed with the trial documentation.

This study does not permit assent from a professional representative or randomisation without prior consent/ assent.

\section{Randomisation}

It is not possible to blind either patients or treating surgeons to when the patient has had surgery or whether they have had surgery. To minimise possible sources of bias randomisation is undertaken centrally by an independent organisation (Centre for Healthcare Randomised Trials, Aberdeen). The allocation is stratified by country group with a minimisation procedure dependent on prognostic criteria and with a random element.

Randomisation must take place within 48 hours of ictus. Randomising clinicians complete a one-page randomisation form before contacting the central 24-hour randomisation service by telephone or web. The randomisation form records demographic and clot characteristics and status at randomisation. This information is required in order to randomise the patient.

During the randomisation process the neurosurgeon is informed of the treatment group the patient is allocated to plus the patient identifier number for the trial. The neurosurgeon records this information on the randomisation form and then faxes the form to the STICH Coordinating centre in Newcastle, UK.

Best medical treatment must begin as soon as possible and continue throughout follow-up, as required. If the patient is randomised to early surgery this should be undertaken within 12 hours of randomisation.

\section{Data Collection and Six Month Follow-up}

The data manager at the STICH Coordinating centre in Newcastle, UK checks the information on the faxed randomisation forms against the information received from the randomisation service and enters the data into an anonymised password protected database. A list of patient names and study numbers is kept in a separate file to ensure patient confidentiality is maintained.
At two weeks after randomisation or at discharge or at death (whichever occurs first), the discharge/2 week form is completed by the responsible neurosurgeon or proxy. This form records:

- The event that triggers the form (i.e. death, transfer or discharge) and the patient's status at that time. - Whether the patient has had surgery (and why if randomised to initial conservative treatment or why not if randomised to early surgery).

- The patient's GCS and localising features for the five days following randomisation.

- The occurrence of any adverse events following randomisation.

- Past medical history and status prior to the ictus.

- Glasgow Coma Score and Glasgow Outcome Scale at discharge from the neurosurgical unit or at two weeks (whichever is earlier).

These data are used by the Data Monitoring Committee to monitor progress of the trial.

The discharge/2 week form together with copies of the randomisation CT scan and the 5-day post randomisation CT scan are sent to the STICH office within two weeks. The preferred method of sending CT scans is in DICOM compatible format. DICOM images (on separate $\mathrm{CDs}$ for the two time points) are sent anonymised with patient identifier. The data manager enters the data into the anonymised password protected database. The CTs are analysed by trained readers blinded to treatment group and patient identity.

Postal follow-up occurs at six months. Structured postal questionnaires are used. They have been translated into the necessary languages. The patient's GP (in the UK) or consultant (outside the UK) is contacted at four months to confirm that the patient is alive, to confirm his/her place of residence and to request completion of the adverse events form. The six-month outcome questionnaire is mailed to the patient or carer at five months and followed with a reminder at six months if needed and telephone follow-up at seven months by "blinded" clerical or nursing staff, if necessary.

In countries where the postal system is problematic the patients are asked to attend a follow-up clinic where the questionnaires will be distributed and collected by an independent researcher. In countries where literacy or language/dialect is problematic an independent blinded interviewer administers the questionnaire. This same methodology was used successfully in STICH.

The aim is to achieve $100 \%$ follow-up and this can be achieved with the full cooperation of the centre investigators. 


\section{Data storage}

All paper copies of questionnaires are kept in locked filing cabinets in a locked office. All computerised data is password protected.

\section{Analysis}

Outcome Measures

Primary Unfavourable outcome will be death or severe disability which will be defined using a prognosis based 8 point Glasgow Outcome Scale/Modified Rankin Scale $[17,19]$.

Secondary Mortality, Rankin, EuroQol, Survival, living arrangements.

\section{Sample size}

Subgroup analysis of the STICH trial demonstrated that for patients with only a lobar haematoma without an intraventricular extension $37 \%$ had a favourable outcome with initial conservative treatment and $49 \%$ had a favourable outcome with early surgery. With a $37 \%$ favourable outcome from conservative treatment a sample size of 566 would be required to show a $12 \%$ benefit from surgery $(2 p<0.05)$ with $80 \%$ power. A sample size of 600 was chosen to allow for some loss to follow up and a small crossover rate.

\section{Blinding}

The multidisciplinary team in the co-ordinating centre and the principal investigators are blinded to the results until after the data set is locked following receipt of the final outcome questionnaire. Only the data manager has access to unblinded data.

\section{Statistical analysis}

Analysis will be on an "intention to treat" basis. The primary analysis will be a simple categorical frequency comparison using the chi-squared test for prognosis based favourable and unfavourable outcomes at six months $[19,23]$. Patients with a good prognosis will be categorised as having a favourable outcome if they achieve good recovery or moderate disability on the Glasgow Outcome scale. Patients with a poor prognosis will be categorised as having a favourable outcome if they achieve good recovery, moderate disability or upper severe disability on the extended Glasgow outcome scale. Logistic regression analysis will be undertaken to adjust for covariates. Secondary outcomes will also be analysed using the prognosis based method as specified in STICH [17].

Any subgroup analyses will be based on tests of interaction. The predefined subgroups include the following:

Age

Volume
Glasgow Coma Score

Time from ictus to randomisation

Severity of neurological deficit

Planned method of haematoma removal

\section{Data and Safety Monitoring}

\section{Roles and responsibilities of Data Monitoring Committee}

The data monitoring committee considers data from interim analyses and reports to the Trial Steering Committee. Interim analyses are strictly confidential and the committee will only recommend stopping the trial early if one or other treatment shows an advantage at a very high significance level.

\section{Roles and Responsibilities}

Roles and responsibilities of Principal Investigators and trial coordinating team

Professor A D Mendelow has overall responsibility for the trial.

Dr B A Gregson is responsible for the overall day-today conduct of the trial including availability of co-ordinating advice in Newcastle.

Professor G D Murray is responsible for overall statistical validity of the trial.

Mr P Mitchell is responsible for recruiting centres and for analysis and publication of results.

Dr A R Gholkar is responsible for the central reading of CT scans.

The data manager is responsible for maintaining computerised databases containing all data related to the trial, for the quality of computerised information, for conducting preliminary analyses and preparing reports for the Data Monitoring Committee, for providing information to the applicants and for preparing monthly newsletters.

The trial secretary is responsible for all trial correspondence in relation to the trial, for sending postal questionnaires and reminders, for the organisation of investigator meetings and travel for monitoring, maintaining telephone and fax communications, preparing quarterly newsletters and publications, and reimbursing centres.

\section{Roles and responsibilities of National Investigators}

In countries with multiple centres one centre investigator fulfils the role of National Investigator. National investigators are responsible for obtaining national ethical approval, for ensuring that documentation is translated from English as required, for identifying suitable centres within their country, for encouraging recruitment and acting as a liaison person between the STICH Coordinating centre team and the centre if required. Each National Neurosurgical Investigator should work with a nominated Physician Champion within their 
country in order to promote the trial to other colleagues.

Roles and responsibilities of Centre Investigators

Each centre agrees to follow the protocol. They provide and update the trial coordinating team with their full address and contact details as necessary. Within each centre there is at least one named collaborator who is responsible for the conduct of the trial in his/her centre and in particular for:

- local ethical applications

- disseminating information about the trial within the centre

- maintaining local trial documentation

- identifying suitable patients

- ensuring all case report forms are completed and returned to the $\mathrm{STICH}$ office in Newcastle expeditiously

- ensuring copies of CT scans are provided to STICH office in Newcastle expeditiously

- ensuring follow-up is obtained in the centre

- attending investigator meetings

- facilitating centre monitoring

- commenting on the final report

Roles and responsibilities of Trial Steering Committee

The Trial Steering Committee provides overall supervision of the trial on behalf of the MRC. It considers progress of the trial, adherence to the protocol, patient safety and consideration of new information. The trial is conducted according to the standards set out in the MRC Guidelines for Good Clinical Practice.

Trial Organisation

STICH Co-ordinating Centre (for all information and queries)

STICH Office, Neurosurgical Trials Unit, Newcastle University, 3-4 Claremont Terrace, Newcastle upon Tyne, NE2 4AE

Website: http://research.ncl.ac.uk/stich/

Email: stich@ncl.ac.uk

Phone: +441912225761

Fax: +44 1912225762

\section{Randomisation Service}

Aberdeen HSRU +44 (0) 1224 551261

https://viis.abdn.ac.uk/HSRU/stich/

\section{Steering Committee}

Professor P Sandercock (Independent Chairman)

Professor G Ford, (Independent Member)

Professor D Barer, (Host Institution Member)

Professor A Strong (Independent Member)

Professor AD Mendelow

Dr BA Gregson
Mr PM Mitchell

Dr AR Gholkar

Professor GD Murray

\author{
Data Monitoring Committee \\ Professor D Hanley (Chairman) \\ Mr DT Hope \\ Dr A Skene \\ Miss Helen Fernandes
}

Trial Management Team

Dr Barbara A Gregson (Trial Director)

Professor A David Mendelow (Chief Investigator)

Dr Elise Rowan (Data Manager 01/05/2008 -)

Dr Alina Andras (Data manager 01/08/2010 -)

Miss Gillian Kenyon (Trial Secretary)

\section{Sponsor}

Newcastle upon Tyne Hospitals NHS Trust (Sponsor No: 3545).

\section{Funding Source}

Medical Research Council (UK) now allocated to Efficacy and Mechanism Evaluation Programme of the National Institutes of Health Research (NIHR):

MRC Grant No: G0501444

EME Number: 09-800-18

This report is independent research funded by the MRC and managed by the NIHR on behalf of the MRCNIHR partnership. The views expressed in this publication are those of the authors and not necessarily those of the MRC, NHS, NIHR or the Department of Health.

\section{List of Abbreviation}

STICH: Surgical Trial in Intracerebral Haemorrhage; STICH II: Surgical Trial in Lobar Intracerebral Haemorrhage; ICH: Intracerebral Haemorrhage; CT: Computerised Tomography; IVH: Intraventricular Haemorrhage; MREC: Multicentre Research Ethics Committee (in the UK); NHS: National Health Service (in the UK); GCS: Glasgow Coma Scale; ml: Millilitres; cm: Centimetres; DICOM: Digital Imaging and Communications in Medicine; GP: General Practitioner; MRC Medical Research Council (UK); EME: Efficacy and Mechanism Evaluation Programme of the National Institutes of Health Research; NIHR: National Institutes of Health Research

\section{Acknowledgements}

The biggest acknowledgment goes to the patients who have given their informed consent to participate in this study and also to the growing number of staff in our participating centres who provide a high level of support and make the research possible. A list of our participating centres is constantly updated on the study website: http://research.ncl.ac.uk/stich/ List of registered recruiting collaborating centres (correct as of $21^{\text {st }}$ March 2011):

ARMENIA - Yerevan State Medical University, Dr Ruben V Fanarjyan AUSTRALIA - Royal Melbourne Hospital, Prof Stephen Davis AUSTRIA - Rudolfstiftung Wein, Vienna, Dr Günther Kleinpeter CANADA - University of Alberta Hospital, Dr Max Findlay CHINA - Tianjin Medical University General Hospital, Prof Shuyuan Yue Beijing Tiantan Hospital, Dr Yuanli Zhao

Huashan Hospital, Fudan University, Shanghai, Dr Ying Mao CZECH REPUBLIC - Central Military Hospital, Prague, Prof Vladimir Benes 
Fakultni Nemocnice, Ostrava, Dr Tomas Krejci University Hospital, Brno, Prof Martin Smrcka St. Anne's University Hospital, Brno, Prof Dr Pavel Cejpak Fakultni Nemocnice, Olomouc, Prof Miroslav Vaverka Regional Hospital, Liberec, Dr Pavel Buchvald EGYPT - Mansoura International Hospital, Dr Abd-Elhafiz Shehab Eldien Zagazig University Hospital, Dr Mohamed Barakat GEORGIA - State Medical Univeristy Clinic, Tbilisi, Prof Alexander Gvelesiani GERMANY - Klinik fur Neurochirurgie, Univ. Magdeburg, Prof Dr Raimund

Firsching

Clemens Hospital, Munster, Prof Dr Abolghassem Sepehrnia

Universitatsklinikum Erlangen, Prof Dr Michael Buchfelder

Klinikum Amberg, Dr Andrea Kleindienst

Heinrich Heine University, Duesseldorf, Dr Daniel Haenggi

University Hospital Heidelberg, Dr Karl Kiening

University Clinic, Muenster, Prof Walter Stummer

Klinikum Kassel, Prof Dr Wolfgang Deinsberger

Ernst Moritz Arndt University, Greifswald, Prof Dr W S Schroeder

University Schleswig-Holstein, Lubeck, Dr Georg Nowak

Helios Klinikum Berlin, Prof Dr med Jurgen Kiwit

Neurochirurgische Klinik, Dessau, Dr Kazimierz Sadowy

Universitatklinikum des Saarlandes, Prof Wolf-Ingo Steudel

Dr. Horst-Schmidt-Kliniken, Wiesbaden, Prof Gerhard Hamman

Klinikum Saarbrucken, Winterberg, Dr S Thomas

Charite - University Medicine, Berlin, Prof Eric Juettler

Universitatsklinikum Jena, Dr Albercht Waschke

Asklepios Klinik Altona, Prof Dr med Uwe Kehler

Diakonieklinikum, Jun-Stilling-Hospital, Siegen, Dr Veit Braun

GREECE - Ippokration General Hospital, Aristotle University, Thessaloniki, Prof

Philippos Tstitsopoulos

Evangelismos Hospital, Mr George Stranjalis

AHEPA General Hospital, Aristotles University, Thessaloniki, Dr Athanasios

Spiliotopoulos

HUNGARY - Pecs University Hospital, Dr Andras Buki

Borsod County and University Teaching Hospital, Dr Jozsef Dobai

INDIA - All India Institute of Medical Sciences, New Delhi, Dr P Sarat

Chandra

BGS Global Hospitals, Bangalore, Dr Shailesh Rao

Christian Medical College and Hospital, Ludhiana, Dr Sarvpreet Singh Grewal

National Neurosciences Centre, Calcutta, Dr K Sridhar

Care Hospital, Visakhapatnam, Dr V P Ramana

Care Hospitals Institute of Neurological Sciences, Hyderabad, Dr T V R K

Murty

Kerala Institute of Medical Sciences, Kerala, Dr Moni Vinod

Acharya Vinoba Bhave Rural Hospital, Maharashtra, Dr Anand Kakani

Kamineni Hospital, Hyderabad, Dr Subodh Raju

Amri Hospitals, Dhakuria, Prof R N Bhattacharya

MM Institute of Medical Sciences and Research, Haryana, Prof Amit Agrawal

Sree Chitra Tirunal Institute for Medical Sciences, Trivandrum, Dr Suresh Nair

ISRAEL - Rambam Hospital, Haifa, Dr Leon Levi

ITALY - University Sapienza, Rome, Prof Robert Delfini

JAPAN - Kanto Medical Center, NTT East Co. Tokyo, Dr Akio Morita

LATVIA - Clinical University Hospital, Gailezers, Dr Kaspars Auslands

Pauls Stradins Clinical University Hospital, Prof Egils Valeinis

LITHUANIA - Klaipeda University Hospital, Prof Antanas Gvazdaitis

Kaunas University Hospital, Antanas Gvazdaitis

MACEDONIA - Clinical Center, Skopje, Prof Dr Kiril Lozance

MALAYSIA - Universiti Sains Malaysia, Kubang Kieran, Prof Dr Jafbi Malin

MEXICO - Instituto Nacional de Neurologia y Neurochirugia, Tlalpan, Dr

Samuel Romero Vargas

Hospital Civil de Guadalajara, Dr Jose Luis Ruiz-Sandoval

MOLDOVA - National Practical and Scientific Centre of Emergency Medicine, Chisinau, Prof Stanislaw Groppa

NEPAL - B. P. Koirala Institute of Health Sciences, Dr Yam Bahadur Roka

B \& B Hospital, Gwarko, Dr Krishna Sharma

NORWAY - St Olavs Hospital, Trondheim University Hospital, Dr Sozaburo Hara

PAKISTAN - Lahore General Hospital, Dr Khalid Mahmood

NW General Hospital and Research Centre, Peshawar, Prof Tariq Khan
POLAND - Poznan University of Medical Sciences, Prof Dr Stanislaw Nowak University Medical School, Bialystok, Prof Zenon Mariak

ROMANIA - Cluj County Emergency Hospital, Prof Ioan Stefan Florian

Country Hospital, Timisoara, Dr Horia Ples

RUSSIA - Novosibirsk Medical University, Prof Alex Krivoshapkin

SAUDI ARABIA - King Khalid University Hospital, Dr Essam A Elgamal

SOUTH KOREA - National Medical Center, Seoul, Prof Dae Hee Han

SPAIN - Hospital Universitario 'Marques de Valdecilla', Santander, Prof

Alfonso Vazquez-Barquero

Hospital Uni. Virgen de las Nieves, Granada, Dr Majed J Katati

Hospital Clinico Universitario, Valladolid, Dr Rosario Sarabia

Hospital de Cruces, Bilbao, Prof Jesus Garibi Undabarra

SRI LANKA - National Hospital of Sri Lanka, Colombo, SRI, Dr H.D.S.

Kularathne

SWEDEN - Akademiska Sjukhuset, Uppsala, Prof Per Enblad

THE NETHERLANDS - University Medical Centre, Groningen, Dr Gert-Jan

Luijckx

TURKEY - University of Istanbul, Prof Orhan Barlas

UK - Ninewell's Hospital, Dundee, Professor M Sam Eljamel

Radcliffe Infirmary, Oxford, Mr Richard Kerr

Hurstwood Park, Haywards Heath, Mr Giles Critchley

James Cook University Hospital, Middlesbrough, Mr Roger Strachan

The National Hospital, London, Mr Laurence Watkins

Aberdeen Royal Infirmary, Dr Pragnesh Bhatt

Southampton University Hospital, Mr Antonio Belli

Addenbrooke's Hospital, Cambridge, Mr Peter Kirkpatrick

Newcastle General Hospital, Professor David Mendelow

Leeds General Infirmary, Mr Jake Timothy

Morriston Hospital, Swansea, Mr Robert Redfern

Salford Royal Infirmary, Mr Andrew King

Preston Royal Infirmary, Mr Aprajay Golash

Western General Hospital, Edinburgh, Dr Rustam al-Shahi Salman

St. George's Hospital (Atkinson Morley's), London, Prof B A Bell

The Walton Centre, Liverpool, Mr Mohsen Javadpour

University Hospital of North Staffordshire, Mr Simon Shaw

Hull Royal Infirmary, Mr Shailendra Achawal

USA - Research Medical Center, Kansas, Dr Iftekar Ahmed

Hartford Hospital, Dr Inam Kureshi

Loyola University Hospital, Chicago, Dr Michael Schneck

Temple University Hospital, Dr Michael Weaver

Central Illinois Neuroscience Foundation, Bloomington IL, Keith Kattner

Georgia Neurosurgical Institute, Dr Arthur Grigorian

Albany Medical Centre, Dr John German

Mayo Clinic, Jacksonville, Dr Benjamin Eidelman

Penn State Hershey Medical Centre, Dr Kevin Cockroft

\section{Author details}

${ }^{1}$ STICH Office, Neurosurgical Trials Unit, Newcastle University, 3-4 Claremont Terrace, Newcastle upon Tyne, NE2 4AE, UK. ${ }^{2}$ Centre for Population Health Sciences, University of Edinburgh Medical School, Teviot Place, Edinburgh EH8 9AG, UK. ${ }^{3}$ Regional Neurosciences Centre, The Newcastle upon Tyne Hospitals NHS Foundation Trust, Royal Victoria Infirmary, Queen Victoria Road, Newcastle upon Tyne, NE1 4LP, UK.

\section{Authors' contributions}

ADM, PMM and BAG are responsible for the conception of the study, protocol design and study coordination. GDM has provided statistical guidance throughout the study and assistance with initial study design. ARG coordinates and provides support with CT scan acquisition and analysis and has also been involved with study management. ENR has been involved with the development of the study. All authors have been involved in the drafting of the manuscript and have approved it prior to submission for publication.

\section{Competing interests}

Professor A David Mendelow is a director of the Newcastle Neurosurgery Foundation Ltd and has received honoraria for attending advisory committee meetings for Codman, Stryker and Novo Nordisc.

Received: 20 April 2011 Accepted: 17 May 2011 Published: 17 May 2011 


\section{References}

1. Bamford J, Sandercock P, Dennis M, Burn J, Warlow C: A prospective-study of acute cerebrovascular disease in the community - the Oxfordshire Community Stroke Project 1981-86.2. Incidence, case fatality rates and overall outcome at one year of cerebral infarction, primary intracerebral and subarachnoid hemorrhage. Journal Of Neurology Neurosurgery And Psychiatry 1990, 53(1):16-22.

2. Broderick J, Brott T, Tomsick T, Tew J, Duldner J, Huster G: Management of intracerebral hemorrhage in a large metropolitan population. Neurosurgery 1994, 34(5):882-7.

3. Fernandes HM, Mendelow AD: Spontaneous intracerebral haemorrhage: a surgical dilemma. Br J Neurosurg 1999, 13(4):389-94.

4. Gregson BA, Mendelow AD, for STICH Investigators: International variations in surgical practice for spontaneous intracerebral haemorrhage. Stroke 2003, 34:2593-8.

5. Siddique MS, Fernandes HM, Wooldridge TD, Fenwick JD, Slomka P, Mendelow AD: Reversible ischemia around intracerebral hemorrhage: a single-photon emission computerized tomography study. J Neurosurg 2002, 96:736-741.

6. McKissock W, Richardson A, Taylor J: Primary Intracerebral haematoma: a controlled trial of surgical and conservative treatment in 180 unselected cases. Lancet 1961, ii:221-6.

7. Auer LM, Diensburger W, Niederkorn K, Gell G, Kleinert R, Schneider G, Holzer P, Bone G, Mokry M, Korner E, Kleinert G, Hanusch S: Endoscopic surgery versus medical treatment for spontaneous intracerebral haematoma. A randomized study. J Neurosurg 1989, 70:530-5.

8. Batjer $\mathrm{HH}$, Reisch JS, Allen BC, Plaizier $L$, Jen Su C: Failure of surgery to improve outcome in hypertensive putaminal haemorrhage. A prospective randomised trial. Arch Neurol 1990, 47:1103-6.

9. Juvela S, Heiskanen O, Poranen A, Valtonen S, Kuurne T, Kaste M, Troupp H: The treatment of spontaneous intracerebral haemorrhage. A prospective randomised trial of surgical and conservative treatment. J Neurosurg 1989, 70:755-8.

10. Chen $X$, Yang $H$, Cheng $Z$ : A prospective randomised trial of surgical and conservative treatment of hypertensive intracerebral haemorrhage. Acta Acad Shanghai Med 1992, 19:237-40.

11. Morgenstern LB, Frankowski RF, Shedden P, Pasteur W, Grotta JC: Surgical treatment for intracerebral hemorrhage (STICH). A single-center, randomised clinical trial. Neurology 1998, 51:1359-63.

12. Zuccarello M, Brott $T$, Derex $L$, Kothari R, Sauerbeck L, Tew J, Van Loveren $H$, Yeh H, Tomsick T, Pancioli A, Khoury J, Broderick J: Early surgical treatment for intracerebral hemorrage. A randomized feasibility study. Stroke 1999, 30(9):1833-9.

13. Cheng XC, Wu JS, Zhao X-P, et al: The randomised multicentric prospective controlled trial in the standard treatment of hypertensive intracerebral hematomas: the comparison of surgical therapeutic outcomes with conservative therapy. Chin J Clin Neurosci 2001, 9:365-8.

14. Hosseini H, Leguerinel C, Hariz M, Melon E, Palfi S, Deck P, Gaston A, Cesaro P, Nguyen JP: Stereotactic aspiration of deep intracerebral haematomas under computed tomographic control, a multicentric prospective randomised trial. Cerebrovasc Dis 2003, 16S4:57.

15. Hattori N, Katayama Y, Maya Y, Gatherer A: Impact of Stereotactic evacuation on activities of daily living during the chronic period following spontaneous Putaminal hemorrhage: a randomized study. Neurosurg 2004, 101:417-20.

16. Teernstra OPM, Evers SMAA, Lodder J, Leffers P, Franke CL, Blaaw G: Stereotactic treatment of intracerebral hematoma by means of a plasminogen activator: a multicenter randomized controlled trial (SICHPA). Stroke 2003, 34:968-74.

17. Mendelow AD, Gregson BA, Fernandes HM, Murray GD, Teasdale GM, Hope DT, Karimi A, Shaw MD, Barer DH, STICH investigators: Early surgery versus initial conservative treatment in patients with spontaneous supratentorial intracerebral haematomas in the International Surgical Trial in Intracerebral Haemorrhage (STICH): a randomised trial. Lancet 2005, 365:387-397.

18. Prasad K, Shrivastava A: Surgery for primary supratentorial intracerebral haemorrhage (Cochrane Review). The Cochrane Library Oxford: Update Software; 2000, 4

19. Mendelow AD, Teasdale GM, Barer D, Fernandes HM, Murray GD, Gregson BA: Outcome assignment in the International Surgical Trial of Intracerebral Haemorrhage. Acta Neurochirurgica 2003, 145:679-681.
20. Mendelow AD, Karmi MZ, Paul KS, Fuller GA, Gillingham FJ: Extradural haematoma: effect of delayed treatment. British Medical Journal 1979, 1(6173):1240-2.

21. Seelig JM, Becker DP, Miller JD, Greenberg RP, Ward JD, Choi SC: Traumatic acute subdural hematoma: major mortality reduction in comatose patients treated within four hours. New England Journal of Medicine 1981, 304:1511-8.

22. Fairbank J, Frost H, Wilson-MacDonald J, Yu LM, Barker K, Collins R: Spine Stabilisation Trial Group. Randomised controlled trial to compare surgical stabilisation of the lumbar spine with an intensive rehabilitation programme for patients with chronic low back pain: the MRC spine stabilisation trial. BMJ 2005, 330(7502):1233-9.

23. Murray GD, Barer D, Choi S, Fernandes H, Gregson B, Lees KR, Maas AIR, Marmarou A, Mendelow AD, Steyerberg EW, Taylor GS, Teasdale GM, Weir CJ: Design and Analysis of Phase III Trials with Ordered Outcome Scales: The Concept of the Sliding Dichotomy. Journal Of Neurotrauma 2005, 22:511-7.

doi:10.1186/1745-6215-12-124

Cite this article as: Mendelow et al:: Surgical Trial in Lobar Intracerebral Haemorrhage (STICH II) Protocol. Trials 2011 12:124.

\section{Submit your next manuscript to BioMed Central and take full advantage of:}

- Convenient online submission

- Thorough peer review

- No space constraints or color figure charges

- Immediate publication on acceptance

- Inclusion in PubMed, CAS, Scopus and Google Scholar

- Research which is freely available for redistribution
Ciomed Central 